\title{
Abordagem conservadora da mão torta ulnar tipo III: Relato de caso*
}

\section{Conservative Approach to Type III Ulnar Club Hand: Case Report}

\author{
Gabriel Chartuni Teixeira Cury ${ }^{10}$ Leonardo Álaf Lucinda de Sá1이 Cler David Oliveira1(1) \\ Nathália Vieira Caires ${ }^{10}$ Arnaldo Gonçalves de Jesus Filho ${ }^{2,3(0)}$ Bruno Gonçalves Schroder e Souza ${ }^{1,4(0)}$
}

1 Faculdade de Ciências Médicas e da Saúde de Juiz de Fora, MG, Brasil

2 Departamento de Ortopedia e Cirurgia da Mão, Hospital Universitário, Universidade Federal de Juiz de Fora, MG, Brasil

${ }^{3}$ Departamento de Ortopedia e Cirurgia da Mão, Santa Casa de Misericórdia de Juiz de Fora, MG, Brasil

${ }^{4}$ Universidade Federal de Juiz de Fora, MG, Brasil

Endereço para correspondência Gabriel Chartuni Teixeira Cury, Faculdade de Ciências Médicas e da Saúde de Juiz de Fora/ Brasil, Rua Maria José Leal, 98, Granville, Juiz de Fora, Minas Gerais, Brasil (e-mail: gabrieljocury@gmail.com).

Rev Bras Ortop

\section{Resumo}

Palavras-chave

- anormalidades musculoesqueléticas

- deformidades congênitas das extremidades superiores

- focomelia

- deformidades congênitas da mão

- ulna/anormalidades
A mão torta ulnar é uma rara afecção de membros superiores, cujo tratamento depende do grau de comprometimento morfológico e funcional, que se correlaciona com a classificação radiográfica de Dobyns, Wood e Bayne. O objetivo deste estudo é relatar um caso de um paciente de 6 anos de idade, do sexo masculino, acompanhado devido a mão torta ulnar do tipo III (aplasia total da ulna). Apesar da dificuldade inicial de manipular objetos e realizar tarefas cotidianas, o tratamento conservador fisioterapêutico propiciou o ganho de força e o desenvolvimento de habilidades funcionais para a vida diária. Concluímos que pacientes com deformidade do tipo III podem ser manejados adequadamente com reabilitação embora necessitem de seguimento ambulatorial até a maturidade esquelética, pois deformidades dinâmicas e novas limitações funcionais podem levar à necessidade de cirurgias corretivas.

Ulnar club hand is a rare upper limbs condition, for which treatment depends on the degree of morphological and functional impairment, correlating with the radiographic classification of Dobyns, Wood, and Bayne. The aim of the present study is to report a case of a 6-year-old male patient, followed up for type III ulnar club hand (total ulnar dysplasia). Despite the initial difficulty of manipulating objects and performing everyday tasks, conservative physical therapy treatment provided strength gain and development of functional skills for daily life. We conclude that patients with type III deformity can be properly managed with rehabilitation although they require outpatient follow-up until skeletal maturity is reached, as dynamic deformities and new functional limitations may lead to need for corrective surgeries.

Trabalho desenvolvido na Faculdade de Ciências Médicas e da Saúde de Juiz de Fora, MG, Brasil

recebido

23 de Dezembro de 2020

aceito

07 de Abril de 2021
DOI https://doi.org/

$10.1055 / \mathrm{s}-0041-1735137$. ISSN 0102-3616. (c) 2021. Sociedade Brasileira de Ortopedia e Traumatologia. All rights reserved.

This is an open access article published by Thieme under the terms of the Creative Commons Attribution-NonDerivative-NonCommercial-License, permitting copying and reproduction so long as the original work is given appropriate credit. Contents may not be used for commercial purposes, or adapted, remixed, transformed or built upon. (https://creativecommons.org/ licenses/by-nc-nd/4.0/)

Thieme Revinter Publicações Ltda., Rua do Matoso 170, Rio de Janeiro, RJ, CEP 20270-135, Brazil 


\section{Introdução}

A mão torta ulnar (MTU) é uma afecção congênita rara. ${ }^{1}$ No Brasil, uma incidência de 1,6\% de MTU foi relatada. ${ }^{2}$ Trata-se de uma das mais raras deformidades congênitas dos membros superiores. $^{2}$

A MTU é uma falha longitudinal na formação da ulna (focomelia), que pode estar total ou parcialmente ausente. ${ }^{3}$ Ela é mais frequente em homens, no lado direito, e unilateral em $70 \%$ dos casos. A forma incompleta é a mais comum. ${ }^{4}$

A deformidade é caracterizada por antebraço curto e curvado para o lado radial, com a mão desviada em sentido ulnar. ${ }^{4}$ Outras anormalidades associadas podem estar presentes. ${ }^{4}$ A função do cotovelo pode estar comprometida em diferentes graus (desde rigidez até aumento da mobilidade, com instabilidade) ${ }^{1,5}$ Em crianças maiores, a luxação da cabeça radial poderá ocorrer. ${ }^{1}$

Dobyns, Wood e Bayne classificam esta afecção em quatro tipos. No tipo I, há hipoplasia da ulna, pequeno desvio ulnar, arqueamento do rádio, e não existe anlage. No tipo II, há aplasia parcial da ulna (terço médio ou distal), a articulação proximal da ulna com o úmero está presente, existe anlage distal, e há luxação da cabeça do rádio. No tipo III, ocorre a aplasia total da ulna, não existe anlage, o rádio é arqueado, e a cabeça do rádio é luxada (com instabilidade do cotovelo). No tipo IV, há sinostose radioumeral e o anlage costuma estar presente. $^{6}$

O objetivo deste estudo é apresentar um caso de MTU tipo III, no qual o desafio foi a escolha do tratamento. Discutimos os elementos que norteiam a indicação terapêutica, os relacionados ao acompanhamento clínico e apresentamos os resultados funcionais após 2 anos de seguimento.

\section{Relato de caso}

Um paciente de 4 anos de idade, do sexo masculino, foi admitido em ambulatório especializado devido a "má formação do membro superior direito (MSD)". O diagnóstico de MTU havia sido realizado por ultrassonografia morfológica e

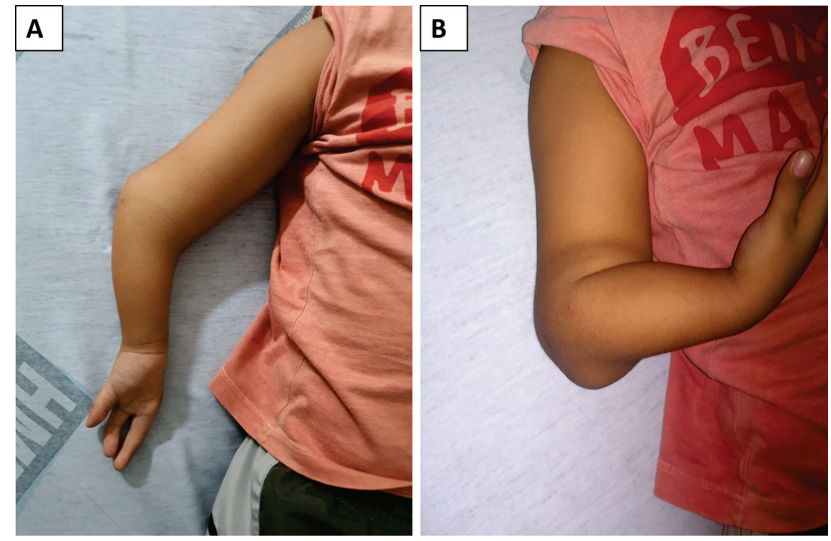

Fig. 1 Ectoscopia da deformidade. (A) Face anterior do membro. (B) Perfil com flexão.

confirmado após o nascimento. Além da MTU, foi diagnosticada agenesia testicular à esquerda. Havia história familiar de atrofia muscular espinhal tipo III (paterna). Este trabalho foi aprovado pelo CEP (CCAE:97048518.9.0000.5103).

Uma deformidade típica de MTU associada à agenesia do quarto e quinto quirodáctilos era evidente ( - Figura 1). Não foi possível identificar formação de barra fibrosa ou cartilaginosa (anlage). Havia importante limitação funcional e dificuldades para manipular objetos além de hipoestesia na borda medial do terceiro dedo.

As radiografias (- Figura 2) evidenciaram ausência completa da ulna, luxação da cabeça do rádio, e ossos do carpo centralizados. Não havia displasia glenoumeral no ombro.

Diante das limitações para a vida diária, o paciente procurou nosso ambulatório onde recebeu indicação de tratamento fisioterápico. O objetivo era otimizar a atividade motora fina e ganho funcional (como usar o celular, se vestir sozinho e colocar os sapatos). O tratamento foi realizado durante 9 meses (1 hora, 3 vezes por semana) (Quadro 1 ).

O resultado da fisioterapia foi mensurado pela escala Goal Attainment Scale (GAS), ${ }^{7}$ que elencou três tarefas consensuais entre os familiares e o terapeuta. Os objetivos foram:
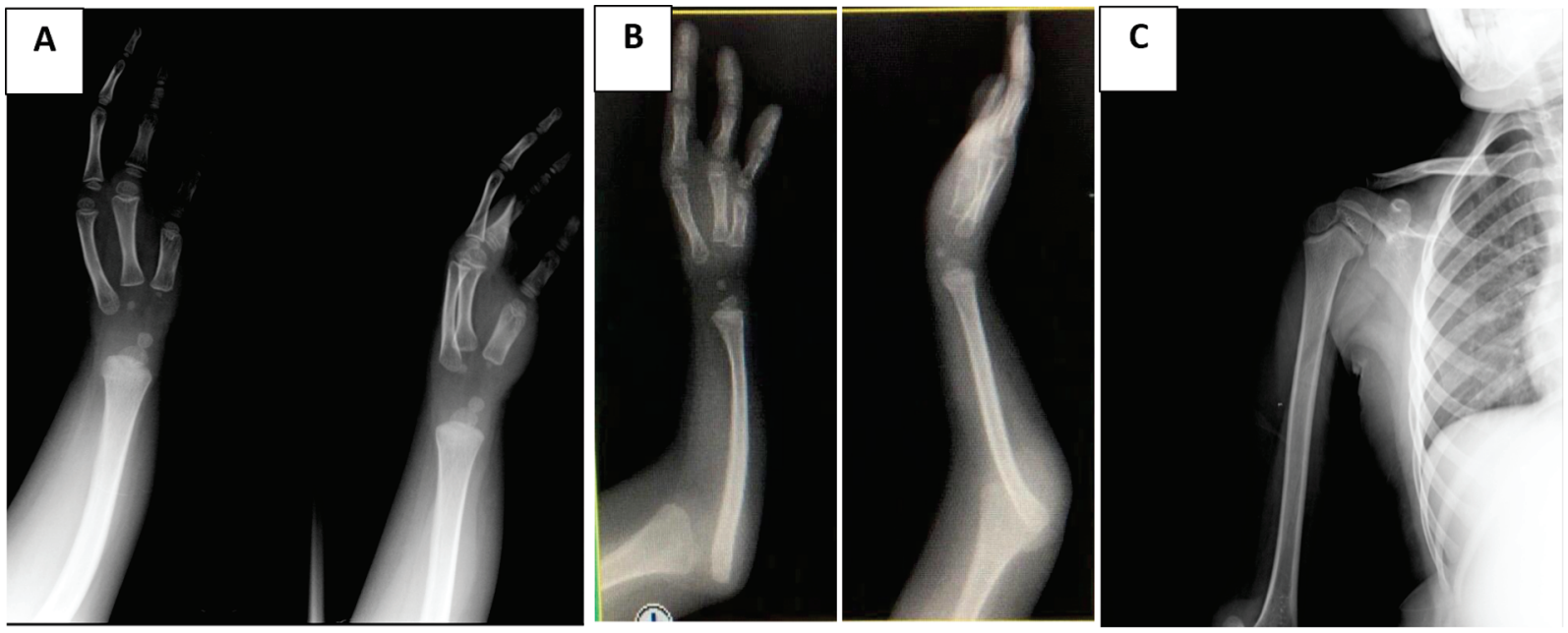

Fig. 2 (A) Mão torta ulnar tipo III de Dobyns, Wood e Bayne - Aplasia total da ulna e ausência de $4^{\circ}$ e $5^{\circ}$ quirodáctilos direitos e seus respectivos metacarpos com relação articular preservada. (B) Luxação da cabeça do rádio. (C) Ausência de deformidades do úmero proximal. 
Quadro 1 Procedimentos fisioterapêuticos realizados pelo paciente durante o período de fisioterapia

\begin{tabular}{|l|}
\hline $\begin{array}{l}\text { 1. Fortalecimento de membro superior direito utilizando caneleira de } 0,5 \mathrm{~kg} \text { associado à atividade de arremessar bola, com uso } \\
\text { de faixa elástica; }\end{array}$ \\
\hline $\begin{array}{l}\text { 2. Fortalecimento das falanges da articulação radiocárpica direita utilizando massa de cera, exercitador para dedos e mãos e } \\
\text { bola de borracha; }\end{array}$ \\
\hline 3. Fortalecimento dos músculos periescapulares associado a atividades lúdicas; \\
\hline 4. Mobilização articular da escapula e glenoumeral; \\
\hline 5. Mobilização osteocinemática da glenoumeral com assistência à escapula; \\
\hline 6. Treino de coordenação motora (aprender a utilizar as falanges de forma individual); \\
\hline 7. Treino de função (amarrar cadarço, vestir roupas, abrir garrafa). \\
\hline
\end{tabular}

melhorar a funcionalidade do MSD com ganho de movimentos finos; aprimorar a preensão palmar; reduzir a discinesia escapular durante a abdução para pegar brinquedo na prateleira. Em 2 meses, o paciente apresentou melhora em 2 tarefas (função do MSD, comparada em vídeo, e melhora da preensão palmar nas tarefas diárias).

Na consulta após a fisioterapia, aplicamos o questionário Disabilities of the Arm, Shoulder and Hand (DASH). ${ }^{8}$ Por ser criança, as questões 7, 8, 12 e 21 não puderam ser aplicadas por abordarem funções típicas dos adultos. Esta modificação nos levou a considerar não o escore absoluto (38,33 pontos), mas sim o seu valor ponderado (46\%), classificando a disfunção como moderada. O Quadro 2 elenca outros achados.

O paciente mantém o acompanhamento após 2 anos, assintomático e com o quadro estável, realiza o movimento de pinça do polegar, apesar de não apresentar função da musculatura de oponência. A musculatura flexora dos três dedos é normal, então o paciente é capaz de brincar com o celular, se vestir independentemente e realizar outras tarefas diárias (-Figura 3). Optamos por não indicar cirurgia neste momento, embora a conduta possa ser revista, principalmente se houver perda da capacidade de pinça do polegar, piora da estabilidade do cotovelo, perda progressiva da função, ou dor.

\section{Discussão}

Nosso caso é ilustrativo da MTU, pois inclui paciente do sexo masculino, de acometimento no lado direito. ${ }^{4}$ Na literatura, a agenesia parcial da ulna (Bayne II) é o fenótipo mais frequente, diferente do nosso caso. ${ }^{1,5,6}$ Não identificamos casos semelhantes ao nosso na literatura nacional.

A agenesia de metacarpos e falanges observada também é reportada na maioria dos estudos. ${ }^{1,5}$ Não identificamos anormalidades musculoesqueléticas concomitantes ou limitação da mobilidade do cotovelo, ao contrário de outros autores. 5,6

O tratamento nos casos de MTU requer abordagem multiprofissional e individualizada. ${ }^{9}$ A maioria das crianças é tratada sem cirurgia, especialmente na MTU unilateral. ${ }^{9}$ Nos casos que necessitam de cirurgia, o objetivo é melhorar a função do membro afetado. ${ }^{9}$

Para avaliar o ganho de função podemos utilizar escalas objetivas (ex.: DASH) ou outras mais subjetivas (ex.: GAS). Embora seja validado, o questionário DASH apresenta desvantagens por envolver um período maior de aplicação e ser específico para adultos. Por isso, o GAS é preferido por nós, por ser mais prático. Ele envolve definir um conjunto de objetivos, especificando uma gama de possíveis resultados

Quadro 2 Avaliação de atividade antes e após a fisioterapia

\begin{tabular}{|l|l|l|}
\hline & Antes da fisioterapia & Após a fisioterapia \\
\hline Função & $\begin{array}{l}\text { Limitação do uso de falange individual em mão direita } \\
\text { Limitação da preensão palmar para realização de tarefas } \\
\text { funcionais } \\
\text { Discinesia escapular durante abdução para pegar objeto em } \\
\text { prateleira }\end{array}$ & $\begin{array}{l}\text { Aquisição da capacidade de usar falange } \\
\text { individual (força muscular: grau 4/5) } \\
\text { Aprimoramento da preensão palmar } \\
\text { Sem melhora observada }\end{array}$ \\
\hline Força & $\begin{array}{l}\text { Hipotonia do membro superior direito } \\
\text { O paciente conseguia vencer gravidade } \\
\text { O paciente não conseguia carregar objeto/brinquedo com } \\
\text { peso com membro superior direito }\end{array}$ & $\begin{array}{l}\text { Mantido } \\
\text { Mantido } \\
\text { Mantido }\end{array}$ \\
\hline $\begin{array}{l}\text { Tarefas } \\
\text { específicas }\end{array}$ & $\begin{array}{l}\text { O paciente conseguia se alimentar sozinho } \\
\text { O paciente conseguia usar o celular } \\
\text { O paciente não conseguia se vestir sozinho } \\
\text { O paciente não conseguia colocar os sapatos } \\
\text { O paciente não conseguia abrir fechaduras } \\
\text { O paciente não conseguia abrir pacote de biscoitos }\end{array}$ & $\begin{array}{l}\text { Mantido } \\
\text { Melhora com uso de falange individual } \\
\text { Aquisição parcial de função** } \\
\text { Aquisição parcial de função } \\
\text { Aquisção de função** } \\
\text { Aquisção de função } \\
\text { Aquisição de função }\end{array}$ \\
\hline
\end{tabular}

*Aquisição parcial de função: Ganho de função com dificuldade e/ou limitação na execução do movimento.

**Aquisição de função: Ganho de função com adequada adaptação à execução do movimento. 


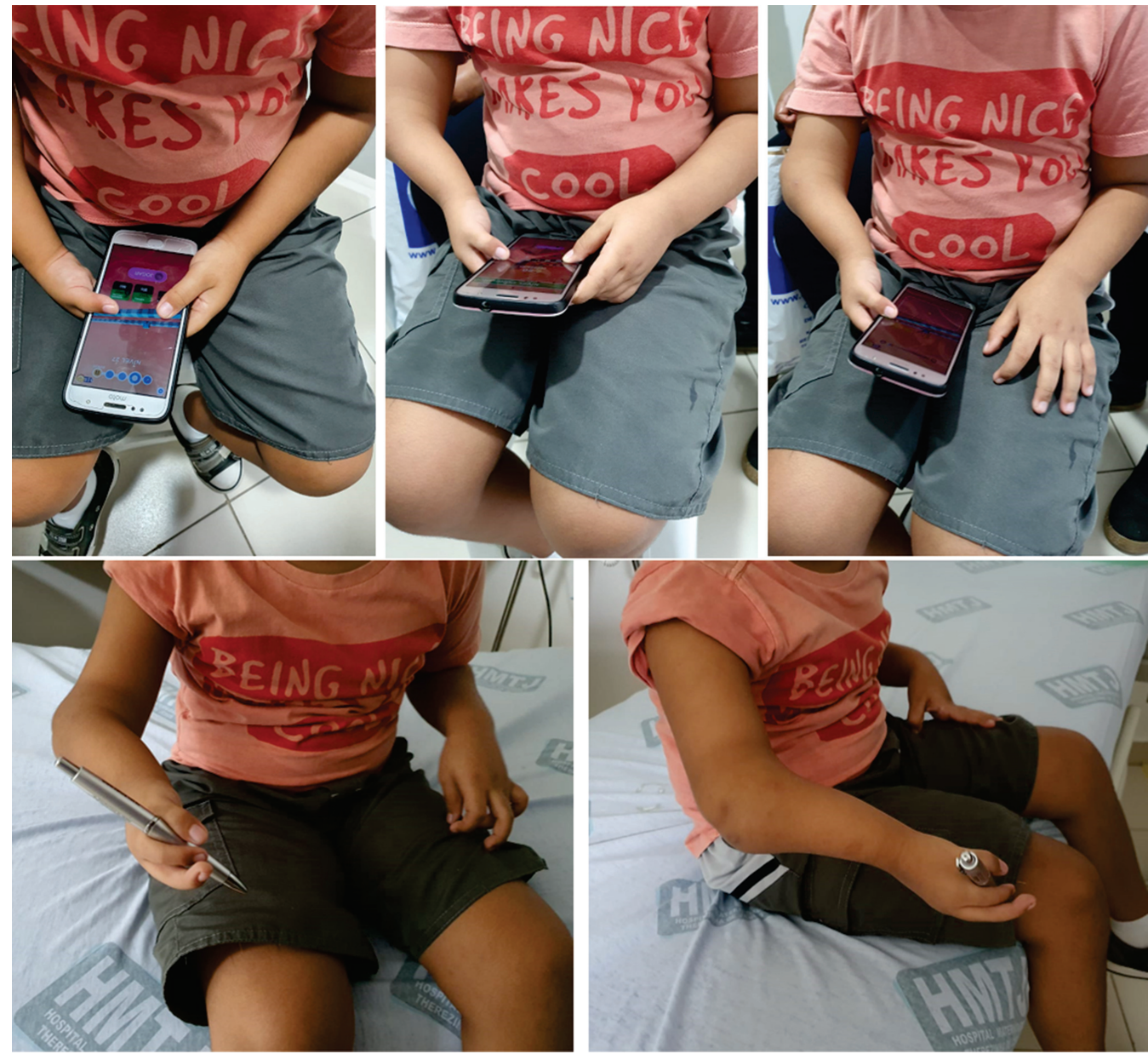

Fig. 3 Imagens ilustrativas da funcionalidade do membro utilizando aparelho eletrônico e demonstrando função de pinça.

para cada objetivo (em uma escala que contém 5 níveis, de -2 a +2 ). É utilizado para avaliar o desempenho após um período de intervenção especificado. ${ }^{7}$

A abordagem cirúrgica nos casos de Bayne II foi advogada por Monteiro e Schachinger. ${ }^{1,5} \mathrm{Em}$ pacientes com Bayne IV, o tratamento conservador é recomendado. ${ }^{10}$ Não identificamos recomendações para Bayne III. Neste caso, optou-se pelo tratamento conservador devido à idade do paciente e boa resposta à fisioterapia com adaptação adequada às atividades do dia a dia.

O prognóstico da MTU dependerá de fatores como: unilateralismo, associação a síndromes, o número de dedos presentes, o grau de atrofia dos membros e a presença de sindactilia. ${ }^{9} \mathrm{Em}$ nosso caso, a função de articulações do cotovelo e punho pode piorar com o crescimento, o que justifica acompanhamentos seriados e eventuais intervenções. ${ }^{1}$ No entanto, o seguimento dos últimos 2 anos evidenciou evolução satisfatória.

\section{Suporte Financeiro}

Não houve suporte financeiro de fontes públicas, comerciais, ou sem fins lucrativos.

\section{Conflito de interesses}

Os autores declaram não haver conflito de interesses.

\section{Referências}

1 Monteiro AV, Chiconelli JR. Deficiência ulnar congênita: conduta de tratamento. Rev Bras Ortop 1997;32(08):637-640

2 Pinto HB, Pais AP, Vitorio SC, Brandão R, Moreira AAD, Molinaro LR. Case Study of Congenital Anomalies of the Upper Limb in Reference Ambulatory Care Facility. Acta Ortop Bras 2018;26 (05):325-327

3 Swanson AB. A classification for congenital limb malformations. J Hand Surg Am 1976;1(01):8-22

4 Panda A, Gamanagatti S, Jana M, Gupta AK. Skeletal dysplasias: A radiographic approach and review of common non-lethal skeletal dysplasias. World J Radiol 2014;6(10):808-825

5 Schachinger F, Girsch W, Farr S. Soft-Tissue Distraction Prior to Single Bone Forearm Surgery in Ulnar Longitudinal Deficiency: A Report of Two Cases. J Hand Surg Asian Pac Vol 2018;23(01): 153-157

6 Bayne LG. Ulnar club hand (Ulnar deficiencies). In: Green DP. Operative hand surgery. New York: Churchill Livingstone; 1993:288-303

7 McDougall J, Wright V. The ICF-CY and Goal Attainment Scaling: benefits of their combined use for pediatric practice. Disabil Rehabil 2009;31(16):1362-1372

8 Orfale AG, Araújo PM, Ferraz MB, Natour J. Translation into Brazilian Portuguese, cultural adaptation and evaluation of the reliability of the Disabilities of the Arm, Shoulder and Hand Questionnaire. Braz J Med Biol Res 2005;38(02):293-302

9 Frantz DH, O'Rahilly R. Ulnar hemimelia. Artif Limbs 1971;15(02): 25-35

10 Elhassan BT, Biafora S, Light T. Clinical manifestations of type IV ulna longitudinal dysplasia. J Hand Surg Am 2007;32(07):1024-1030 